\title{
Chapitre 3 Principes de la sûreté pour les réacteurs à eau sous pression du parc français
}

\subsection{Introduction}

Il ne s'agit pas dans ce chapitre d'exposer de manière exhaustive les principes et la pratique de la sûreté nucléaire dans les installations nucléaires françaises (le lecteur pourra se référer à l'ouvrage de J. Libmann pour une présentation du sujet [1]), mais d'illustrer les deux approches de sûreté complémentaires que sont l'approche déterministe et l'approche probabiliste par la présentation d'un concept fort de la première, la défense en profondeur, et d'une description générale des études probabilistes de sûreté (EPS) relevant de la seconde.

Les installations nucléaires, notamment celles destinées à la production d'électricité, présentent des risques spécifiques liés à la présence de quantités plus ou moins importantes de substances radioactives qui peuvent conduire à l'exposition aux rayonnements ionisants d'individus, de populations ainsi que de l'environnement.

La sûreté nucléaire est constituée d'un ensemble de dispositions techniques et d'organisation prises à tous les stades de la vie d'une installation (conception, construction et mise en service, exploitation, arrêt définitif et démantèlement) pour protéger les travailleurs, les personnes du public et l'environnement contre les effets des substances radioactives. Il s'agit :

- d'assurer le fonctionnement normal des installations sans exposition excessive des travailleurs et sans rejets excessifs de radioactivité dans les effluents radioactifs ; 
- de prévenir les incidents et les accidents ;

- de limiter les conséquences des incidents et des accidents qui surviendraient malgré les dispositions de prévention mises en œuvre.

Le confinement des substances radioactives est réalisé par l'interposition de « barrières » entre ces substances et les personnes. Schématiquement, pour un réacteur à eau sous pression (REP) du type de ceux qui sont exploités en France, on distingue en fonctionnement trois barrières successives entre les substances radioactives et l'environnement: les gaines des crayons combustibles, le circuit primaire de refroidissement et l'enceinte de confinement. La meilleure étanchéité possible de ces barrières dans les différentes situations de fonctionnement normal et accidentel doit être recherchée à la conception. Cependant, même en fonctionnement normal, les barrières ne sont généralement pas parfaitement étanches: des ruptures de gaines, des fuites du circuit primaire et de l'enceinte de confinement d'importance limitée peuvent se produire?

Il faut souligner ici le cas particulier des tubes des générateurs de vapeur des REP qui font partie des deuxième et troisième barrières dans la mesure où la rupture d'un tel tube peut entraîner l'ouverture des soupapes de sûreté du générateur de vapeur correspondant, créant ainsi un bipasse du confinement.

La défense en profondeur, présentée au paragraphe suivant, vise à assurer les fonctions fondamentales de sûreté que sont la maîtrise de la réactivité, le refroidissement du combustible irradié et le confinement proprement dit des substances radioactives ; ces fonctions de sûreté sont nécessaires pour maintenir l'efficacité de l'ensemble des barrières.

\subsection{Concept de défense en profondeur}

Le concept de défense en profondeur a été introduit dans le domaine de la sûreté nucléaire au début des années 1970. Il se concrétise pour les installations nucléaires par la mise en place d'une série de niveaux de défense reposant sur les caractéristiques intrinsèques de l'installation, des dispositions matérielles et des procédures destinées à prévenir les accidents puis, en cas d'échec de la prévention, à en limiter les conséquences. La défense en profondeur est un concept qui s'applique à tous les stades de la vie d'une installation, de la conception au démantèlement.

La mise en œuvre effective du concept de défense en profondeur évolue au cours du temps, en tenant compte du retour d'expérience du fonctionnement des installations, et notamment des incidents et des accidents survenus dans les installations, de manière à construire une défense toujours plus efficace.

Pour les réacteurs en exploitation, la défense en profondeur est aujourd'hui structurée en cinq niveaux (voir le rapport INSAG-10 [2] et le tableau 3.1), visant à prévenir

1. Les défauts d'étanchéité en fonctionnement normal doivent cependant permettre de respecter les spécifications techniques d'exploitation. 
l'apparition et à limiter les conséquences de défaillances techniques, humaines et organisationnelles. Les différents niveaux de la défense en profondeur s'appliquent dans les différents états de l'installation, du fonctionnement normal jusqu'aux accidents de fusion du cœur. À chaque niveau de la défense en profondeur, sauf pour le niveau 5, correspondent des dispositions visant à prévenir l'évolution vers des situations plus graves.

Lors de la conception des réacteurs actuellement en exploitation, la défense en profondeur ne comprenait que trois niveaux.

\section{Niveau 1: prévention des anomalies de fonctionnement et des défaillances des systèmes}

La prévention des anomalies de fonctionnement et des défaillances des composants, équipements et systèmes suppose une conception prudente (dimensionnement avec des marges) et une haute qualité dans la fabrication et l'exploitation des composants, équipements et systèmes. Ce niveau correspond au domaine de fonctionnement normal de l'installation avec des règles générales et des procédures d'exploitation qui visent au maintien de la tranche dans son domaine de fonctionnement normal.

\section{Niveau 2 : détection des défaillances et maîtrise des anomalies de fonctionnement}

À ce niveau, correspondent les moyens et les dispositions visant à maîtriser les anomalies de fonctionnement, ce qui suppose une surveillance permettant d'assurer la détection des défaillances. Citons ici les automatismes, les systèmes de régulation et de contrôle permettant de ramener l'installation dans son domaine de fonctionnement normal. Ces systèmes visent à arrêter une évolution anormale des paramètres de l'installation.

\section{Niveau 3 : maîtrise des accidents (jusqu'aux « accidents de dimensionnement »)}

Les deux premiers niveaux de la défense en profondeur permettent de réduire les risques de défaillance de l'installation. Il est néanmoins fait l'hypothèse que des accidents peuvent survenir au cours de l'exploitation du réacteur. Les accidents considérés à ce niveau résultent d'un événement initiateur unique (par exemple, la défaillance d'un élément important pour assurer une fonction fondamentale de sûreté - maîtrise de la réactivité, refroidissement du combustible nucléaire, confinement des substances radioactives). Des moyens permettant de limiter les conséquences de tels accidents et d'assurer les fonctions fondamentales de sûreté sont mis en place : à ce niveau de la défense en profondeur correspond la mise en place des systèmes de sauvegarde qui assurent, pour les accidents (jusqu'aux accidents considérés pour le dimensionnement de l'installation), l'intégrité de la structure du cœur et la limitation des rejets dans l'environnement. À ce niveau correspond également la définition des procédures de conduite en situation accidentelle. 
Après l'accident de Three Mile Island, le concept de défense en profondeur a été étendu pour traiter des accidents qui n'avaient pas été explicitement considérés lors de la conception des installations. En particulier, les enseignements tirés des premières études probabilistes de sûreté (§ 3.3) et de l'accident de TMI-2 survenu en 1979 aux États-Unis (§ 7.1) ont montré la nécessité de considérer les accidents résultant de défaillances multiples et les accidents conduisant à la fusion du cœur. Ces développements ont conduit à la définition d'un niveau supplémentaire dans la défense en profondeur.

\section{Niveau 4 : maîtrise des accidents graves}

Ce niveau de la défense en profondeur correspond aux procédures et matériels mis en œuvre pour faire face à des situations non traitées par les trois premiers niveaux de la défense en profondeur ; il s'agit des accidents qui peuvent éventuellement conduire à la fusion du cœur du réacteur. Au niveau 4, on cherche donc d'une part à éviter que des accidents puissent évoluer vers une fusion du cœur, d'autre part à limiter les rejets à l'extérieur du site par le maintien de la fonction de confinement des substances radioactives dans le cas où une fusion du cœur surviendrait néanmoins.

À ce niveau de la défense en profondeur, correspondent les procédures ultimes (procédures $U$ ) et les moyens matériels associés ( $\$ 2.5 .2$ ), des moyens matériels spécifiques (recombineurs d'hydrogène par exemple), le guide d'intervention en accident grave (GIAG) et le plan d'urgence interne (PUI) de l'installation. Le plan d'urgence interne est établi et mis en œuvre par l'exploitant. Lorsque le PUI est mis en œuvre, les équipes de crise de l'installation sont mobilisées dans l'objectif d'arrêter la progression de l'accident et d'éviter le rejet de substances radioactives. Le PUI a également pour objet, d'une part la protection du personnel travaillant sur le site en cas d'incident ou d'accident, d'autre part la limitation des conséquences de l'accident à l'extérieur du site.

\section{Niveau 5 : limitation des conséquences radiologiques en cas de rejets de substances radioactives}

Malgré l'ensemble des dispositions décrites précédemment, des rejets radioactifs sont susceptibles de se produire. Des dispositions complémentaires, relevant des pouvoirs publics, sont alors mises en œuvre pour protéger les populations, le personnel travaillant sur le site et les biens des conséquences de ces rejets.

Les mesures envisageables de protection des populations à l'égard des rejets radioactifs sont l'évacuation, la mise à l'abri dans des locaux en dur, l'ingestion de comprimés d'iodure de potassium, des restrictions de consommation des denrées alimentaires. À ce niveau correspondent notamment les plans particuliers d'intervention (PPI) établis pour chaque site. Le PPI est mis en œuvre par les pouvoirs publics et fixe l'organisation des secours en vue de limiter l'exposition des populations aux rayonnements en cas de rejet. 
Tableau 3.1. Les différents niveaux de la défense en profondeur.

\begin{tabular}{|c|c|c|c|}
\hline Niveau & Objectif & Principales mesures & $\begin{array}{c}\text { État correspondant } \\
\text { de l'installation }\end{array}$ \\
\hline 1 & $\begin{array}{l}\text { Prévention des anomalies de } \\
\text { fonctionnement et des défail- } \\
\text { lances des systèmes }\end{array}$ & $\begin{array}{l}\text { Conception prudente (dimen- } \\
\text { sionnement avec marges) et } \\
\text { qualité de la construction et } \\
\text { de l'exploitation de l'instal- } \\
\text { lation }\end{array}$ & $\begin{array}{l}\text { Fonctionnement } \\
\text { normal }\end{array}$ \\
\hline 2 & $\begin{array}{l}\text { Détection des défaillances } \\
\text { et maîtrise des anomalies } \\
\text { de fonctionnement }\end{array}$ & $\begin{array}{l}\text { Systèmes de régulation, de } \\
\text { protection, de contrôle (main- } \\
\text { tien de l'installation dans son } \\
\text { domaine de fonctionnement } \\
\text { normal) et de surveillance } \\
\text { (prévention des défaillances) }\end{array}$ & $\begin{array}{l}\text { Anomalies de fonc- } \\
\text { tionnement ou } \\
\text { défaillances }\end{array}$ \\
\hline 3 & $\begin{array}{l}\text { Maîtrise des accidents } \\
\text { (jusqu'aux « accidents } \\
\text { de dimensionnement ») }\end{array}$ & $\begin{array}{l}\text { Systèmes de sauvegarde et } \\
\text { procédures accidentelles }\end{array}$ & $\begin{array}{l}\text { Accidents jusqu'aux } \\
\text { « accidents de } \\
\text { dimensionnement » } \\
\text { (événement initiateur } \\
\text { unique) }\end{array}$ \\
\hline 4 & $\begin{array}{l}\text { Maîtrise des accidents graves, } \\
\text { prévention de la progression } \\
\text { des accidents et limitation } \\
\text { des conséquences }\end{array}$ & $\begin{array}{l}\text { Mesures complémentaires et } \\
\text { management de l'accident } \\
\text { (procédures U et moyens } \\
\text { matériels associés, GIAG, PUI) }\end{array}$ & $\begin{array}{l}\text { Défaillances multiples } \\
\text { Accidents de fusion } \\
\text { du cœur }\end{array}$ \\
\hline 5 & $\begin{array}{l}\text { Limitation des conséquences } \\
\text { radiologiques en cas de rejet } \\
\text { de substances radioactives }\end{array}$ & $\begin{array}{l}\text { Gestion de la crise radiolo- } \\
\text { gique hors du site (PPI) }\end{array}$ & Accidents avec rejet \\
\hline
\end{tabular}

Pour les réacteurs de génération III, les accidents résultant de défaillances multiples et les accidents de fusion du cœur sont considérés dans la conception initiale de ces réacteurs, ce qui représente une évolution majeure dans l'étendue des situations accidentelles pour lesquelles des mesures de prévention et de limitation des conséquences doivent être prévues à l'origine. Même si les dispositions prévues pour ces réacteurs ne sont pas toutes applicables en pratique aux réacteurs de génération II, elles peuvent aider à identifier des améliorations de sûreté pour les réacteurs actuellement en exploitation et à renforcer la défense en profondeur de ces réacteurs.

\subsection{Apports de l'approche probabiliste}

Des études probabilistes de sûreté (EPS) ont été développées à partir des années 1960 pour les centrales électronucléaires. Le rapport Rasmussen [3], publié en 1975 aux ÉtatsUnis, qui visait à comparer les risques encourus par la population du fait des réacteurs nucléaires avec les autres risques industriels et les risques naturels, a démontré l'intérêt d'une analyse probabiliste pour apprécier les risques des réacteurs nucléaires. Toutes les centrales nucléaires de puissance en exploitation dans le monde ont depuis fait l'objet d'études probabilistes de sûreté (EPS).

Les EPS complètent les analyses déterministes classiques. Les EPS permettent en effet de procéder à une investigation systématique des nombreuses possibilités de 
cumuls et d'enchaînements d'événements pouvant constituer des scénarios accidentels. Elles se composent d'un ensemble d'analyses techniques permettant d'apprécier les risques d'une installation en termes de fréquences des accidents, par exemple la fusion du cœur, et de leurs conséquences. Elles permettent une vue globale de la sûreté des réacteurs, intégrant aussi bien la tenue des équipements que le comportement des opérateurs. Elles peuvent mettre en évidence des sujets pour lesquels des évolutions tant de la conception que de l'exploitation peuvent être étudiées, voire être jugées nécessaires.

Il est classique de distinguer trois grands types d'EPS selon les conséquences étudiées :

- EPS de niveau 1 : elles permettent d'identifier les séquences pouvant mener à la fusion du cœur et de quantifier leurs fréquences ;

- EPS de niveau 2 : elles permettent d'évaluer la nature, l'importance et les fréquences des rejets de substances radioactives hors de l'enceinte de confinement ;

- EPS de niveau 3 : elles permettent d'évaluer les probabilités des conséquences sur les personnes du public en termes dosimétriques ou en termes de contamination (voire en termes de fréquences de cancers ou d'autres effets sur la santé).

Le rapport Rasmussen [3] est le premier exemple d'une EPS de niveau 3. Comme nous le verrons au paragraphe 4.3.2, ce rapport reste encore aujourd'hui un rapport de référence pour l'approche de la gestion des accidents avec fusion du cœur. Les études probabilistes de sûreté réalisées jusqu'à présent en France pour les tranches de $900 \mathrm{MWe}$, les tranches de 1300 et 1450 MWe et le réacteur EPR, sont des études de niveau 1 et de niveau 2 [4-9] (voir également les $\S 4.2$ et 4.4). Elles sont réalisées par EDF et l'IRSN, les études réalisées par EDF étant les études de référence. Elles sont réactualisées par EDF et par l'IRSN, notamment lors des réexamens de sûreté, pour tenir compte de l'évolution des connaissances ainsi que de l'expérience d'exploitation. Elles sont utilisées pour l'évaluation de sûreté des réacteurs à eau sous pression dans les conditions indiquées dans la règle fondamentale de sûreté $n^{\circ} 2002$ 01 [10]. Les résultats des EPS de niveau 1 ainsi que la méthode et les applications des EPS de niveau 2 réalisées par l'IRSN sont décrits dans le paragraphe 4.4.

Par rapport aux études du même type conduites à l'étranger, les études de niveau 1 et de niveau 2 françaises bénéficient de la standardisation des tranches des réacteurs français qui permet d'apprécier la fiabilité des matériels et les probabilités de certains événements initiateurs sur des bases statistiques plus importantes. De plus, les études françaises traitent tous les états du réacteur : fonctionnement à pleine puissance, fonctionnement à puissance intermédiaire, réacteur à l'arrêt pour intervention. Les EPS françaises ont ainsi mis en évidence que les états d'arrêt d'un réacteur conduisent à une part importante de la probabilité de fusion du cœur, compte tenu des configurations spécifiques d'exploitation dans ces états, et notamment du moindre degré d'automatisation des actions de sauvegarde. Ces résultats ont entraîné des améliorations d'exploitation (spécifications techniques, procédures) et de conception (mise en place d'alarmes, d'automatismes).

Les mêmes EPS ont également permis d'apprécier plus quantitativement l'intérêt des dispositions prises pour améliorer la conduite accidentelle. Les EPS de niveau 2 sont également utilisées pour évaluer les dispositions des guides d'intervention en accident grave que les opérateurs sur site devraient mettre en œuvre dans de telles situations, notamment pour assurer autant que possible le confinement des substances radioactives [11-13]. 
Les EPS présentent cependant certaines limitations dues aux incertitudes qui leur sont associées et cela implique de prendre des précautions dans l'interprétation de leurs résultats et leur utilisation dans les processus de prise de décision. Les EPS françaises existantes ne sont en effet pas exhaustives en termes de couverture, la prise en compte des agressions internes et externes n'étant que très partielle. Par ailleurs, des incertitudes résultent des données quantitatives d'entrée et des simplifications et hypothèses retenues pour conduire l'étude [14]. On peut citer, de manière non exhaustive, les incertitudes associées aux choix de regroupement des événements initiateurs, aux choix de scénarios pour les calculs thermohydrauliques et neutroniques en support, à la modélisation des phénomènes physiques et des actions humaines, à l'estimation de la fiabilité des logiciels et des équipements, ou encore au choix des arbres d'événements (choix des événements, ordre chronologique) et des logiciels de quantification probabiliste (voir le $§ 4.4$ pour plus de détail).

Les EPS de sûreté globales sont donc utilisées pour conforter ou compléter les analyses de sûreté déterministes classiques, à la fois pour apprécier de façon plus quantitative le niveau de sûreté des tranches françaises et pour constituer des outils d'analyse de ces tranches. Autant les comparaisons des résultats des EPS à des critères chiffrés sont à manier avec précaution, compte tenu des incertitudes évoquées plus haut, autant la démarche probabiliste permet de déterminer des faiblesses relatives des tranches examinées et d'apprécier par exemple, en relatif, l'intérêt de modifications de conception ou d'exploitation.

\subsection{Conclusion}

Les approches de sûreté déterministe et probabiliste constituent un ensemble qui concourt à la prévention et à la limitation des conséquences des accidents, notamment des accidents graves avec fusion du cœur, et donc à assurer un niveau de sûreté élevé des installations nucléaires. L'évolution de cet ensemble est continue et il convient de toujours garder à l'esprit l'interaction permanente entre le niveau de sûreté des installations et l'état des connaissances auquel participent la recherche, présentée pour le domaine des accidents de fusion du cœur au chapitre 5 de cet ouvrage, les études approfondies (comme les EPS) présentées au paragraphe 4.4, l'expérience d'exploitation, et l'analyse des incidents et des accidents.

Les installations nucléaires françaises ont notamment fait l'objet en 2011 suite à l'accident de Fukushima Daïchi, d'évaluations complémentaires de sûreté (ECS) qui ont porté pour les réacteurs de puissance en priorité sur cinq points : les risques d'inondation, les risques sismiques, les pertes d'alimentation électrique, les pertes de la « source froide » et la gestion opérationnelle des situations accidentelles. Ces évaluations visent à apprécier la robustesse des réacteurs français pour faire face à des évènements externes extrêmes et à compléter les mesures de sûreté existantes pour renforcer cette robustesse.

En particulier, des efforts sont en cours à l'IRSN afin d'élargir le champ d'application des EPS en y intégrant les connaissances récentes obtenues par la recherche, en traitant les agressions telles que les inondations et les séismes et en tenant compte du retour d'expérience du fonctionnement des installations ; ces efforts visent à une meilleure appréciation des risques des réacteurs de puissance et des dispositions prises pour la conduite accidentelle. 


\section{Références}

[1] J. Libmann, Éléments de sûreté nucléaire, EDP Sciences, collection IPSN, 1996.

[2] International Nuclear Safety Advisory Group Report, INSAG-10, IAEA Report, Vienna, 1996.

[3] N. Rasmussen et al., Reactor safety study, WASH-1400, Washington DC, US NRC, 1975.

[4] A. Ellia-Hervy, F. Corenwinder, J.-M. Lanore, V. Sorel, Les Études Probabilistes de Sûreté de niveau 1 : les méthodes, les connaissances utilisées, les résultats, Revue Générale Nucléaire 1, 12-16, 2003.

[5] E. Kalalo, D. Brenot, Rôle et limites des EPS, Revue Contrôle 155, 39-42, 2003.

[6] A. Dubreuil-Chambardel, G. Body, V. Sorel, Qu'est-ce qu'une étude probabiliste de sûreté de niveau 2 ? Exemple de la troisième visite décennale des REP $900 \mathrm{MWe}$, Revue Générale Nucléaire 1, 88-92, 2010.

[7] E. Raimond, Apport des études probabilistes de sûreté de niveau 2 dans l'analyse de sûreté - Point de vue de l'IRSN, Revue Générale Nucléaire 1, 99-105, 2010.

[8] E. Raimond, N. Rahni, K. Chevalier-Jabet, T. Durin, L'EPS de niveau 2 pour les REP 900 : du développement aux enseignements de l'étude, IRSN, Rapport scientifique et technique, 2008.

[9] E. Raimond, C. Caroli, B. Chaumont, Status of IRSN level 2 PSA, CSNI/WG Risk Workshop, Cologne, Allemagne, 2004.

[10] Règle fondamentale de sûreté $n^{\circ}$ 2002-01 - Développement et utilisation des études probabilistes de sûreté pour les réacteurs nucléaires à eau sous pression, document ASN (2002); http://www.asn.fr/index.php/Les-actions-de-lASN/La-reglementation/Regles-fondamentales-de-surete-et-guides-de-l-ASN/ Guides-de-l-ASN-et-RFS-relatives-aux-REP/RFS-2002-1-du-26-12-2002.

[11] E. Raimond, B. Laurent, N. Rahni, K. Chevalier-Jabet, T. Durin, Application des EPS de niveau 2 et des techniques de fiabilité dynamique à la validation des guides d'intervention en cas d'accident grave, IRSN, Rapport scientifique et technique 2007.

[12] E. Raimond, T. Durin, B. Laurent, K. Chevalier-Jabet, Level 2 PSA: a dynamic event tree approach to validate PWR severe accident management guidelines, Conference PSA2008, Knoxville, USA, 2008.

[13] E. Raimond, K. Chevalier-Jabet, F. Pichereau, Link between level 2 PSA and off-site emergency preparedness, Conference PSAM8, New Orleans, USA, 2006.

[14] E. Raimond, N. Rahni, M. Villermain, Method implemented by the IRSN for the evaluation of uncertainties in level 2 PSA, Workshop on the evaluation of uncertainties in relation to severe accidents and level 2 PSA, Cadarache, France, 2005. 